Pensamiento Crítico Vol. 20 N$^{\circ}$ 1, pp. 65-74

\title{
Estructura de capital, valor de la empresa y rentabilidad: el caso de las empresas del sector alimentos en el Perú: 2009-2013
}

Capital Structure, Value of the company and Profitability: The case of the Food Sector companies in Peru: 2009-2013

[Recepción: Marzo de 2015/ Conformidad: Mayo 2015]

Gaby Cortez Cortez*

\section{RESUMEN}

El trabajo se propuso a través de la especificación de un modelo probar la consistencia del objetivo de la creación de valor de las empresas, considerando tres determinantes principales: el crecimiento, la rentabilidad y el apalancamiento. Los resultados preliminares confirman las hipótesis generales planteadas en la investigación.

Las variables más relevantes en el trabajo están referidas a los pasivos financieros de las empresas y al capital accionario de las mismas que en su conjunto y en combinación forman la estructura de capital. El grado en que la empresa se

* Gaby Cortez Cortez: Profesora Principal de la FCE-UNMSM. Economista por la UNMSM. Magíster en Economía por la UNMSM. Estudios Doctorales en Gestión Económica Empresarial por la UNMSM. Associate in Applied Science in Passaic County College, USA. 


\title{
Pensamiento Crítico Vol. 20. NoI
}

endeuda determinará su estructura de capital. Si bien no existe una receta para encontrar el grado óptimo de esta estructura de capital, el intercambio entre deuda y capital nos permitirá conocer los cambios en el riesgo que asumen las empresas y su rendimiento esperado. En términos teóricos más deuda aumenta el riesgo y se espera un rendimiento mayor. Si las empresas aumentan su deuda asumen mayor riesgo produciendo una caída en el precio de la acción y esperan un rendimiento mayor. Estos planteamientos teóricos unidos a las hipótesis planteadas son parte del cuerpo del trabajo.

La muestra de empresas materia de estudio corresponden al sector alimentos de la economía peruana destacando las empresas azucareras (Casa Grande, Laredo y Paramonga ), sector lácteos (Gloria y Laive), sector conservas de pescado (Austral Group), y alimentos en diversos sectores (Alicorp) que cotizan sus acciones en la Bolsa de Valores de Lima.

Palabras clave: Estructura de capital, valor de la empresa, rentabilidad.

Clasificación JEL: G12

\begin{abstract}
The work has proposed trough the specification of a model to test the consistency of the objective of creating corporate value considering three main determinants: growth, profitability and leverage. Preliminary results confirm the general hypotheses in the research.

The most important variables in the work are related to the company's financial liabilities and their capital stock that together and combined forms the capital structure.

The extent to which the firm borrows determines its capital structure. While there is no recipe for finding the optimal degree of the capital structure, the exchange between debt and equity offers insight into the changes in the risk facing the company and its expected return. In theoretical terms more debt increases the risk and higher return is expected. If companies increase their debt they assume greater risk leading a drop in the share price and higher returns are expected. These theoretical approaches linked to the established hypotheses are part of the body work.
\end{abstract}




\section{Gaby Cortez Cortez}

The sample of companies studied are in the food sector of the Peruvian economy, highlighting the sugar companies (Casa Grande, Laredo and Paramonga), dairy sector (Gloria and Laive), canned fish sector (Austral Group), and food in various sectors (Alicorp); all listed its shares on the Lima Stock Exchange.

Keywords: Capital structure, value of the companies, profitability.

\section{Introducción}

El trabajo parte de una premisa teórica y de gestión empresarial fundamental, que se refiere a la creación de valor para los accionistas. Al respecto, la literatura basada en la creación de valor de las empresas, busca las motivaciones y los determinantes principales de creación de valor para la empresa. Existen diversos planteamientos que dan mayor o menor relevancia a ciertos componentes de la gestión empresarial y de mercado sobre los determinantes de creación de valor y que se pueden resumir como principales: el crecimiento, la rentabilidad y el apalancamiento de la empresas (ver Liow, 2010). El éxito de la gestión empresarial de la creación de valor es que sus resultados permitan asegurar en el tiempo que el valor de mercado del capital invertido por los accionistas supere su valor contable.

En el plano de la observación empírica es difícil conocer las diversas motivaciones individuales de gestión de las empresas para la creación de valor por cuanto responden a las múltiples características y riesgos que asumen las empresas para la creación de valor y que están enmarcadas por las condiciones propias del producto, su elaboración, la productividad, la ubicación geográfica, el mercado y demandas específicas.

Esta complejidad no escapa a la industria del sector alimentos de la economía peruana, donde se puede observar empresas pequeñas muy frágiles en el desarrollo de sus actividades, y empresas medianas generalmente relacionadas o vinculadas a empresas con gran capacidad en el manejo de fuentes de financiamiento y dominio de mercado. Esto determina que la percepción del riesgo de cada una de ellas sea diferente en cuanto a sus metas y resultados. 


\section{Pensamiento Crítico Vol. 20. NoI}

\section{Revisión de la literatura}

Existe una variedad de trabajos elaborados sobre el tema y para diversos países del mundo de los cuales hemos considerado aquellos más afines a la investigación:

El trabajo de S. Antwi sobre Ghana titulado "Capital Structure and Firm Value: Empirical Evidence from Ghana" (2012) nos señala que en una economía emergente como Ghana, tanto el capital social como la deuda de largo plazo, componentes de la estructura de capital, son los principales determinantes del valor de una empresa. Esta investigación ha examinado la teoría de la estructura de capital y su relación con el valor de la empresa en el contexto de Ghana, tomando 34 empresas que cotizan en la bolsa de valores de dicho país. Los autores sostienen que todas las otras teorías, excepto la teoría de Modigliani y Miller (MM) (1958), han intentado resolver el rompecabezas de la estructura de capital enunciado en las proposiciones de MM (1958). Se indica que cada una de estas teorías relajan las condiciones bajo las cuales se deriva el teorema de MM (1.958). Basándose en estos planteamientos teóricos fundamentales y en los resultados de su estudio, afirman de manera concluyente que: las decisiones de la estructura de capital tienen varias implicancias y una de ellas es su efecto sobre el valor de la empresa. Se recomienda que las empresas comparen siempre el beneficio marginal de la utilización de la deuda a largo plazo con el costo marginal de la deuda a largo plazo, antes de usarla para financiar sus operaciones. Finalmente, se muestra en este trabajo que la deuda a largo plazo tiene un impacto positivo en el valor de las empresas al igual que el capital social.

Chen, Yinghong y Hammes, Klaus en su trabajo "Capital Structure" (2003), a través de un análisis de datos de panel para 7 países: Canadá, Dinamarca, Alemania, Italia, Suecia, Inglaterra y los Estados Unidos encontraron que el tamaño de la empresa, la rentabilidad, la tangibilidad, el ratio de valor de capital de mercado, el ratio de valor del capital contable, y el ratio de deuda contable tienen un impacto significativo en la elección de la estructura de capital de las empresas.

Raj S. Dhankar y Ajit S. Bora en su trabajo realizado para la India titulado "Cost of Capital, Optimal Capital Structure and Value of Firm: An Empirical Study of Indian Companies" (1996) plantean la interrogante respecto a que si ¿Los cambios en la estructura de capital afectan el valor de una empresa?. De los resultados de su investigación se desprende que la estructura de capital afecta el costo de capital, la

ganancia neta, el beneficio por acción, la razón de pagos de dividendos y la posición de 


\section{Gaby Cortez Cortez}

liquidez de las empresas. De otro lado, no encuentran una relación significativa entre los cambios en la estructura de capital y el valor de la empresa, a nivel micro. Esto estaría explicado por el hecho de que el valor de una empresa es afectado por múltiples factores y la estructura de capital es solo una de ellas. Muchos de estos factores tales como la reputación de los promotores, la gerencia de la empresa, las condiciones económicas y políticas, no son medibles porque son factores cualitativos por naturaleza. Debido a este problema, sus efectos no pueden ser segregados, y por lo tanto, no se puede establecer una relación exacta entre los cambios en la estructura de capital y el valor de una empresa a nivel micro. Sin embargo, a nivel macro la relación fue estadísticamente significativa a un nivel de 5 por ciento de significación con un $r$ de 0.706 .

Kim Hiang Liow en su trabajo realizado para el sector inmobiliario de Singapur titulado "Firm Value, Growth, Profitability and Capital Structure of Listed Real Estate Companies: An International Perspective" (2010) considera como principales determinantes del valor de las empresas de bienes inmobiliarios el crecimiento, la rentabilidad y el apalancamiento. Los resultados encontrados en esta investigación demostraron que generalmente las empresas con mayor tamaño tenían una mejor valoración de mercado en relación a su valor contable. Por lo general estas empresas se consideran más rentables y más propensas al efecto positivo del apalancamiento lo que contribuye al crecimiento rentable de largo plazo. Este trabajo encontró respuestas similares para otros países de la región asiática.

Eugene Fama y Kenneth R. French en su trabajo titulado "The cross-section of expected stock returns" (1992) plantean que dos variables de fácil medición como el tamaño y el valor expresados a través de la relación del valor de Mercado y del valor en libros del capital (book to market) pueden capturar la variación del rendimiento medio de los valores asociados a las variables tamaño, apalancamiento, valor del capital (capital-book-to market) y ganancias en los precios.

\section{Planteamiento del estudio}

El análisis de las características y formas de gestión empresarial se han centrado principalmente en los determinantes de la creación de valor empresarial. La rentabilidad, el crecimiento y el apalancamiento financiero han sido adoptados como los determinantes principales en el presente trabajo, desarrollando un modelo con un número reducido de 


\section{Pensamiento Crítico Vol. 20. NoI}

variables explicativas que las usualmente utilizadas en trabajos previos. Existen razones para tal decisión. La primera, no disponer de información para la elaboración de series para determinadas variables que se incluyen en algunos modelos de creación de valor. Segundo, un número reducido de variables permite ponderar con mayor precisión la incidencia de cada una de estas variables sobre la variable independiente.

En nuestro modelo se ha considerado como variable explicada el ratio valor de mercado sobre el valor en libros de las acciones individuales (VM/VL) que es utilizado en muchos trabajos como una medida de la rentabilidad de las acciones y oportunidades de crecimiento de una empresa y que sostienen que el objetivo máximo de gestión de una empresa en su estrategia de creación de valor es lograr el mayor VM, dado un VL.

Se han utilizado cuatro variables explicativas que a nuestro entender reflejan mejor el movimiento de la variable explicada, teniendo en cuenta la información disponible, la realidad empresarial de la economía peruana, y un mercado de acciones pequeño.

La primera, tamaño de la empresa (VM) es una variable que explica el retorno de los activos. Esta variable se utiliza como una variable de control y se mide como el logaritmo de la capitalización de mercado (capitalización bursátil)

La segunda variable margen (MRG) tiene dos componentes: a) ROE como un indicador de la rentabilidad del capital propio y b) el costo del patrimonio ( $\mathrm{Cp}$ ) que es el rendimiento mínimo que los accionistas esperan dado el riesgo esperado para su inversión. El costo del patrimonio (Cp.) se mide como el recíproco del rendimiento de las acciones individuales ( precio / beneficio de la acción). El diferencial positivo, es decir, ROE - Cp. > 0 implica un crecimiento rentable y por lo tanto mayor será el valor de la empresa.

La tercera variable el ratio de deuda $(\mathrm{OBF})$ medida como el ratio la deuda a largo plazo sobre los activos totales. Se ha considerado dentro de la deuda a largo plazo la parte corriente de dicha deuda.

Finalmente, la variable tangibilidad del activo fijo (ACF) que se define como el ratio de los activos fijos actuales de la empresa sobre el Activo total. 


\section{Gaby Cortez Cortez}

\section{Especificación del modelo}

El modelo comprende una ecuación con una variable dependiente (VM/VL) y cuatro variables independientes:

$(\mathrm{VM} / \mathrm{VL})=\mathrm{f}(\mathrm{VM}, \mathrm{MRG}, \mathrm{OBF}, \mathrm{ACF})$

Expresado en términos de ecuación:

$$
(\mathrm{VM} / \mathrm{VL})=\mathrm{B} 0+\mathrm{B} 1 \mathrm{VM}+\mathrm{B} 2 \mathrm{MRG}+\mathrm{B} 3 \mathrm{OBF}+\mathrm{B} 4 \mathrm{CAF}
$$

Se ha utilizado Mínimos Cuadrados Ordinarios para la regresión econométrica de la ecuación de cada una de las empresas seleccionadas con los siguientes resultados resumidos en el cuadro 1 .

Cuadro 1: Resultado de las regresiones de empresas seleccionadas

\begin{tabular}{cccccccc}
\hline & \multicolumn{7}{c}{ Empresas } \\
\cline { 2 - 7 } Parámetros & Alicorp & Austral & Cgrande & Gloria & Laredo & Paramonga & Laive \\
\hline $\boldsymbol{\beta}_{\mathbf{1}}$ & 0.170473 & 0.126663 & 0.334191 & 0.887613 & 0.426023 & 0.236008 & 0.106474 \\
$\boldsymbol{\beta}_{\mathbf{2}}$ & 6.509676 & 1.277359 & 0.333643 & 0.400783 & 1.239678 & 0.099906 & 0.735508 \\
$\boldsymbol{\beta}_{\mathbf{3}}$ & 3.498407 & -1.557218 & 3.354347 & -8.387711 & 1.054261 & 1.472485 & 0.537642 \\
$\boldsymbol{\beta}_{\mathbf{4}}$ & -3.218681 & -0.920645 & -3.019971 & -3.347414 & -3.401464 & -2.192837 & 1.072461 \\
$\mathbf{R}^{2}$ & 0.927141 & 0.222840 & 0.916818 & 0.694590 & 0.792726 & 0.173270 & 0.470217 \\
$\mathbf{D W}$ & 1.506311 & 1.382892 & 2.344954 & 1.631254 & 2.246199 & 1.263892 & 2.419312 \\
\hline
\end{tabular}

Fuente: SMV - BVL

Elaboración: Gaby Cortez

Los resultados de las regresiones conforme a lo esperado muestran signos positivos en los parámetros de la variable tamaño (VM) y el ratio (VM/VL). Sin embargo, esta relación positiva no implica magnitudes directamente proporcionales entre las magnitudes de estas variables.

En cuanto a los resultados que muestran los parámetros de la variable margen (MRG) se puede señalar una relación positiva entre esta variable y el ratio (VM/VL), diferenciándose los parámetros en la magnitud de sus cifras. Por ejemplo, el parámetro de la variable margen (VM/VL) más alto corresponde a la empresa Alicorp (6.509676), correspondiendo a su vez al porcentaje más alto del ratio (VM/VL) ascendente a 1.739. En contraste, la cifra más baja de este parámetro (0.099906) corresponde a la Empresa Agroindustrial Paramonga con la penúltima cifra más baja (0.610) del ratio (VM/VL) de la muestra. 


\section{Pensamiento Crítico Vol. 20. NoI}

Los parámetros correspondientes a la variable obligaciones financieras (OBF) muestran signos positivos y negativos. La afectación de los signos negativos en los parámetros corresponde generalmente a las empresas con más altos porcentajes de endeudamiento. Por ejemplo, al parámetro de la variable OBF de la empresa Gloria (-8.387711) le corresponde porcentajes de deuda de 23.6 por ciento; de igual forma al parámetro de la empresa conservera Austral Group (-1.557218) le corresponde un porcentaje de deuda de 33.2 por ciento. Estos montos pueden ser considerados como riesgosos para el sistema. El efecto

Finalmente, los parámetros de la variable tangibilidad del activo Fijo (ACF) muestran signo negativo de acuerdo a lo esperado, afectando a la variable dependiente (VM/VL). Las empresas con mayores montos de activo fijo físicos podrían tener bajos niveles de beneficios debido al alto apalancamiento operativo, lo cual tiene incidencia en la variable VM/VL.

\section{Interpretación de datos}

En términos generales los resultados de las regresiones muestran un comportamiento esperado, conforme a la teoría relativa al valor de la empresa representado por el ratio (VM/VL), así como a los signos de los parámetros de las variables independientes propuestas en el modelo.

La variable dependiente tamaño (VM) se encuentra positivamente relacionada con la variable independiente representada por ratio (VM/VL) difiriendo en la cifra de los parámetros de las regresiones de las empresas propuestas en el estudio. No se ha podido establecer que al mayor o menor tamaño de la empresa (capitalización bursátil) le corresponda incrementos igualmente proporcionales en el ratio (VM/VL)

Las variables margen (MRG) y ratio de deuda $(\mathrm{OBF})$ se encuentran positivamente relacionadas con la variable (VM/VL), excepto los parámetros de la variable ratio de deuda (OBF) correspondiente a las empresas Gloria y Austral Group que muestran signos negativos.

La variable Tangibilidad de activo fijo $(\mathrm{ACF})$ se encuentra relacionada con signo negativo con la variable independiente (VM/VL). 


\section{Gaby Cortez Cortez}

\section{Conclusiones}

Los resultados de las regresiones de las empresas consideradas en el trabajo nos muestran que los parámetros de las ecuaciones no tienen la misma ponderación para cada una de éstas, lo que nos permite asumir que existen diferencias en los modelos de gestión de empresarial para lograr los objetivos de creación de valor.

La empresa Gloria tiene la cifra más alta (0.887613) en el parámetro de la variable tamaño (VM). En sentido contrario, la empresa Laive muestra la cifra más baja en el parámetro (0.106474), que a su vez muestra el monto más pequeño de capitalización bursátil (9'438,000 de nuevos soles) del conjunto de empresas. Siguen con cantidades menores las empresas agroindustriales :Laredo (0.426023), Casa Grande (0.334191) y Paramonga (0.236008). La empresa Alicorp muestra un parámetro de 0.170473 , a su vez con el monto más alto de capitalización bursátil (2,634'826,919 nuevos soles). En penúltimo lugar se ubica la empresa Austral Group con un parámetro de 0.126663, y el tercer lugar en cuanto magnitud de su capitalización bursátil (454'273,952 nuevos soles). Estas cifras nos permite concluir que el tamaño (VM) tiene un efecto positivo sobre el ratio (VM/VL), sin embargo, la magnitud de esta variable no parece no tener efecto proporcional en cuanto a la magnitud del parámetro. Podríamos asumir más bien que este efecto responde a la percepción que el mercado tiene sobre el valor de cada una de estas empresas.

El segundo parámetro de la variable margen (MRG) se muestra más alto para la empresa Alicorp (6.509976), siendo el más bajo para la empresa Agroindustrial Paramonga (0.099906). En una escala de mayor a menor podemos ubicar para el resto de las empresas a: Austral Group (1.277359), Laredo (1.239678), Laive (0.735508), Casa Grande (0.333643) y Gloria (0.400783). La magnitud positiva de este parámetro nos muestra el crecimiento rentable de la empresa, y por lo tanto magnitudes mayores implican un mayor valor de la empresa (VM/VL).

El tercer parámetro ratio de deuda (OBF) muestra signos positivos y negativos. Para unas empresas el endeudamiento tiene un efecto positivo sobre la rentabilidad y el valor de la empresa. En esta lista se puede ubicar en una escala de mayor a menor a: Alicorp (3.498407), Casa Grande (3.354347), Paramonga (1.472485), Laredo (1.054261) y Laive (0.537642). En cuanto a las empresas con parámetros con signo 


\section{Pensamiento Crítico Vol. 20. NoI}

negativo tenemos a Gloria (-8.387711) y Austral Group (-1.557218). Al respecto podemos señalar que la empresa Gloria muestra altos índices de endeudamiento que el mercado percibe como riesgosos y por lo tanto afectan la cotización de sus acciones. En cuanto a la empresa Austral Group revela periodos de dificultades económicas con niveles altos de endeudamiento considerados riesgosos para el mercado.

El cuarto y último parámetro se encuentra el ratio tangibilidad de activo fijo (ACF) es un parámetro que afecta de manera negativa el valor de la empresa. Las inversiones en activos fijos materiales incrementan los costos operativos de las empresas generalmente asociados a mayores índices de deuda. En una escala de mayor a menor ubicamos a: Laredo (-3.401464), Gloria (-3.347414), Alicorp (-3.21868), Casa Grande (-3.019971), Paramonga (-2.192837) y Austral Group (-0.920645). La excepción es la empresa Laive que muestra signo positivo en su parámetro asociado a un menor índice de activos físicos y bajos niveles de endeudamiento.

\section{Referencias bibliográficas}

Antwi, Samuel and Ebenezer Fiifi Emire Atta Mills, Xican, Zhao. (2012). Capital Structure and Firm Value: Empirical Evidence from Ghana. International Journal of Business and Social Science.

Chen, Yinghong,and Hammes, Klaus. (2003). Capital Structure. Theories and empirical results. Department of Economics Gothemburg University.

Dhankar, Raj.S. and. Boora. (1996). Ajit S. Cost of Capital, Optimal Structure, and Value of Firm: An Empirical Study of Indian Companies. Faculty of Management Studies. University of Delhi.

Fama, E.F., and French, K. (1992). The cross-section of expected stock returns. Journal of Finance.

Hiang Liow, Kim. (2010). Firm Value, growth, profitability and capital structure of listed real estate companies: an international perspective. Journal of Property Resarch. National University of Singapur. 